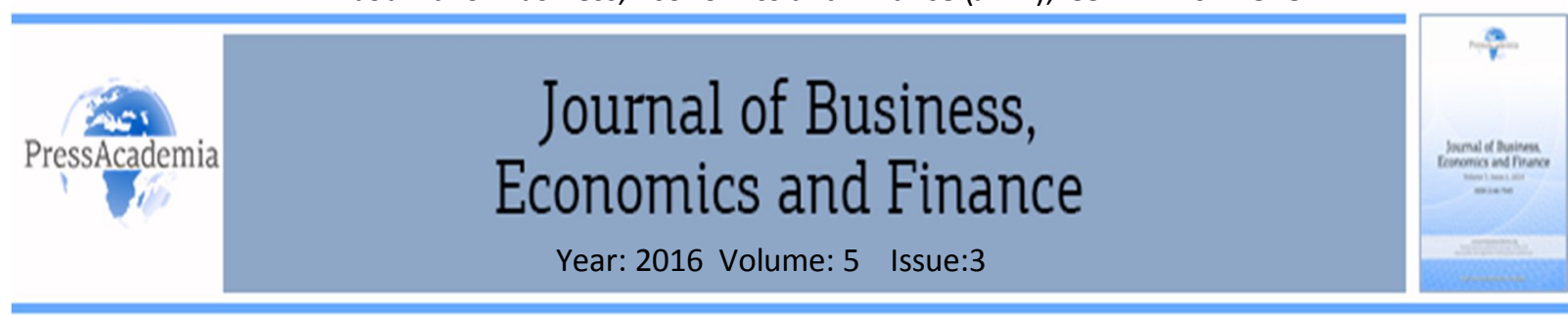

\title{
TRADE CREDIT IN ITALY: FINANCING TO SELL
}

\section{DOI: 10.17261/Pressacademia.2016321975}

\author{
Candida Bussoli ${ }^{1}$, Claudio Giannotti ${ }^{2}$ \\ ${ }^{1}$ LUM Jean Monnet University. bussoli@lum.it \\ 2LUMSA University. c.giannotti@lumsa.it
}

\begin{abstract}
The analysis considers Italian SMEs and investigates, over the years 2006-2011, the existence of interdependencies between trade credit policy and trade debt policy and the co-existence of conditions of complementarity and substitutability between trade credit and other financing sources. Linear regression models on a yearly basis are used and these models are put under observation for six years. The paper shows that there are interdependencies between trade credit policy and trade debt policy; trade credit can be a complementary and substitute source of financing to bank loans; conditions of substitutability and complementarity can also be observed by considering separately firms involved in agricultural or industrial activities and firms that perform services.
\end{abstract}

Keywords: Trade credit, trade debt, bank-firm relationship, SMEs, intercompany financing

JEL Classification: G10, G20, G21

\section{INTRODUCTION}

Italian firms characterise themselves by an elevated recourse in self-financing, bank debts - especially over short periods - multiple exposures (Banca d'Italia 2010, 2011, 2012) and intercompany financing. Many reasons lead to the use of trade credit which can be traced to real and financing functions. Real functions refer to credit offers which support the selling policy. Trade credit may be a way to strengthen client relationships, to guarantee product quality and to allow discrimination of prices; ultimately, this represents a possible response to the variability of demand and a way to improve the profitability of firms. From a financing point of view, trade credit may represent an alternative form of financing in the short term. This paper focuses on the relevance of transactional and financing motivations, as well as the dual function of substitutability or complementarity of trade credit in comparison to other forms of third party financing.

The empirical analysis is carried out on Italian SMEs during the period immediately before and after the outbreak of the financial crisis (2006-2011). This study contributes to reference literature for various reasons. Firstly, Italy represents an important case to study the determinants and the implications of trade credit, as it is characterised by an elevated practice of trade credit, much higher than other European countries. Secondly, this study was carried out over a six-year period, before and during the economic and financial crisis, allowing us to observe the financial function of trade credit even in times of crisis. Thirdly the survey investigates the possibility that recourse in trade credits can be influenced by the enterprise's characteristics (size and age) and by their territoriality and business sector. For these purposes the Italian economic system represents an

\footnotetext{
${ }^{1}$ This paper is the result of a joint effort and a continuous exchange of ideas among the two authors. The individual parts are attributed as follows: Candida Bussoli has written Literature Review, Data and Methodology, Findings and Discussions; Claudio Giannotti has written Introduction and Conclusion.
} 
important contest at an international level, as it consists of SMEs characterised by different economic development conditions, also depending on their location: northern and central regions compared to southern regions and islands. Finally, in literature the financial reasons for recourse in trade credit are usually dealt with debtor firms. This study also takes into consideration creditor firms, and the overall net position of enterprises: the difference between trade receivables and payables that contributes to the financial needs for working capital. The results of this study confirm that there are interdependencies between trade credit policy and trade debt policy. Trade credit, also available in periods of crisis, can be a complementary and substitute source of financing to bank loans. Conditions of substitutability and complementarity can also be observed by considering separately firms involved in agricultural or industrial activities and firms that perform services.

The paper is organized as follows: the second section gives us a brief review of the literature that leads to the research hypotheses; the third section illustrates the methodology and the sample, and the fourth discusses the results. The last paragraph sets forth brief conclusive assessments and the implications of the studied phenomenon.

\section{LITERATURE REVIEW}

Trade credit is the financing between enterprises, resulting from the granting of agreed deferred payments to the client by the supplier. Through the provision of trade credit, the seller forgoes an immediate cash flow, for real-type motivations, in the hope to set up, expand or consolidate relations with customers.

The main aim is to begin a relationship with potential clients and to expand relationships with existing clients: trade credit can be a useful support tool for sales policies and a way to undertake and consolidate relationships with customers, thanks to the product quality guarantee and price discrimination. (Lee and Stowe 1993; Long Malitz and Ravid 1993; Schwartz and Whitcomb 1978, 1979). Another aim is represented by the consolidation of business relations (Emery 1987). Apart from real-type motivations, recourse in trade credit also has financial motivations. Literature weighs on the relevance of the financial reasons for the use of trade credit, distinguishing the transactional and financial components. The transactional element refers to trade credit as a synchronisation tool between receipts and payments instead of using the money, for a better forecast of cash flow, and treasury management planning in case of unexpected payments. From this point of view Schwartz (1974) and Ferris (1981) claim that the demand and supply of trade credit for transactional reasons explain the short-term and very short-term components of trade credit, which reduces the transaction costs and the liquidity buffers for precautionary reasons.

Other studies on the transactional costs hypothesise a positive relationship between demand variability and supplier loan (Emery 1987; Long, Malitz and Ravid 1993). Long, Malitz and Ravid (1993) demonstrated that enterprises which face variable demand make more use of trade credit than enterprises that have steady demand. Transactional reasons refer to the use of trade credit which synchronizes receipts and payments and proves to be convenient both for the seller and the purchaser: the short-term duration and the granting of discounts, when paying by cash, distinguish this operation from trade credit for financial reasons. A survey carried out by Banca d'Italia (Finaldi Russo and Leva 2004) demonstrated that transactional reasons are predominant for enterprises which make a more intense use of trade credit. Financial reasons are of lower importance and the influence of financial reasons affects trade debt much less than real-type motivations. The importance of financial reasons in intercompany financing operations is justified by the increased availability of trade debt compared to other sources of funding, the convenience of trade debts being less onerous compared to other sources, the higher ductility of the financing source which, on one hand, has no complex contractual formulas and on the other hand may have an extended duration with no extra costs. Inaccurate information on the financial system and the presence of weak financial institutions (Fisman, Love 2001) and also weak legal recovery rules (Carmignani 2004) may determine substitutability or complementarity between trade credit and bank financing. Imperfections in the financial markets may determine the rationing of bank financing (Schwartz 1974), which has a greater influence on the financing of opaque or young enterprises (Huyghebaert 2006). The rationing of bank financing may lead to recourse in trade credit as a fall-back determined by the insufficiency and inadequacy of sources of finance from third parties or banks (Duca 1996; Jaffe and Stiglitz 1990; Petersen and Rajan 1997). Complementarity conditions and an extended recourse in trade credit is noted when nonfinancial enterprises know how to monitor the credit capacity of a firm, and they are able to transfer funds, 
borrowing from intermediaries and granting deferred payments to other firms, otherwise rationed for reasons of misinformation (Demirguc, Kunt and Maksimovic 2001). In this case, trade credit can also be a signal: information that financial intermediaries receive from relationships with non-financial firms granting deferred payments can mitigate misinformation that may cause problems for opaque enterprises and may decrease the conditions of credit rationing. Opaque firms that are predominantly small in size can be subject to credit rationing (Stiglitz and Weiss 1981) and can turn to trade credit as an alternative source of financing. Trade credit, obtained according to the assessment made by the credit provider in respect of the creditworthiness of the firms to be financed, increases the good reputation of creditors and allows them to earn easy access to bank credit. Alphonse Ducret and Severin (2004) therefore claim that trade credit can be replaceable, but also complementary to bank debts. The substitutability and complementarity between trade credit and bank credit can occur in the economic-financial system with the prevailing real motivations for recourse in trade credit. The importance of financial motivations may also appear where larger sized firms that do not have to worry about limited access to the financial market, in terms of funds and costs, act as intermediaries, offering trade credit to smaller firms which face greater difficulties in accessing credit market (Emery, 1984: Mian and Smith, 1992; Schwartz, 1974). The financial motivation to use trade credit implies that the provision of trade receivables could have a positive impact on the profitability of larger sized firms, in this way consolidating their relationship with clients. An even more positive impact is expected for small and medium-sized enterprises as the higher percentage of trade payables may represent the availability of intercompany financing even in situations of opacity and credit rationing by banks or other intermediaries.

It may be therefore useful to investigate the importance of financial determiners of intercompany credit and the existence of relationships of substitutability and complementarity between intercompany credit and other sources of financing by third parties. Small and medium-sized enterprises can be subject to conditions of sale from suppliers that have a larger market share: the offer of trade credit, imposed by the exploitation of the market power of suppliers, may determine the adoption of a balancing strategy, financing the supply of trade credit with trade debt. To investigate the financial reasons for recourse in intercompany credit, it is necessary to acknowledge the existence of an interdependent relationship between trade credit policy and trade debt policy, the existence of relationships of substitutability and complementarity between intercompany credit and other sources of financing by third parties and the influence of the business sector.

As claimed in literature and for the above reasons, the following research hypotheses are formulated:

HP1: A strict interdependence relationship exists between trade credit policies and trade debt policies for small and medium-sized enterprises.

HP2: Conditions of substitutability and complementarity coexist between intercompany credit and financing from banks or third parties for small and medium sized enterprises.

HP3: Conditions of substitutability and complementarity coexist between intercompany credit and financing from banks or third parties for small and medium sized enterprises that belong to industry and services sectors.

\section{DATA AND METHODOLOGY}

In coherence with literature, to verify the research hypotheses, linear regression models on a yearly basis are used and these models are put under observation for six years. The variables used for the analysis are presented in Table No. 1.

To verify the research hypotheses the following models are used:

$$
\begin{aligned}
& Y_{i k}=\alpha+\beta_{1} \text { inbanel }_{i}+\beta_{2} \text { inbanol }_{i}+\beta_{3} \text { inalen }_{i}+\beta_{4} \text { inalol }_{i}+\beta_{5} \text { cosden }_{i}+\beta_{6} \text { ros }_{i}+\beta_{7} \text { roe }_{i} \\
& +\beta_{8} \text { curr }_{i}+\beta_{9} \text { solven }_{i}+\beta_{10} \text { ldim }_{i}+\beta_{11} \text { logeta }_{i}+\beta_{12} \text { darea }_{i}+\varepsilon \\
& Y_{i k}=\alpha+\beta_{1} \text { inbanel }_{i}+\beta_{2} \text { inbanol }_{i}+\beta_{3} \text { inalen }_{i}+\beta_{4} \text { inalol }_{i}+\beta_{5} \text { cosden }_{i}+\beta_{6} \text { ros }_{i}+\beta_{7} \text { roe }_{i} \\
& +\beta_{8} \text { curr }_{i}+\beta_{9} \text { solven }_{i}+\beta_{10} \text { ldim }_{i}+\beta_{11} \text { logeta }_{i}+\beta_{12} \text { darea }_{i}+\beta_{13} \text { varspec }_{i}+\varepsilon
\end{aligned}
$$


Table 1: Regression Variables

\begin{tabular}{|c|c|c|}
\hline NAME & DESCRIPTION & MEASURE \\
\hline crclta & Incidence of trade receivables & $\begin{array}{l}\text { Ratio of the sum of accounts receivable in the short and } \\
\text { medium term and total assets }\end{array}$ \\
\hline deforta & Incidence of trade payables & $\begin{array}{l}\text { Ratio of the sum of trade payables in the short and } \\
\text { medium term and total assets }\end{array}$ \\
\hline crmerta & Incidence of net mercantile credit & $\begin{array}{l}\text { Ratio of the difference between trade receivables and } \\
\text { trade payables and total assets }\end{array}$ \\
\hline ros & Return on Sales & Ratio of operating income and sales revenues \\
\hline roe & Return on Equity & Ratio of net income and shareholders' equity \\
\hline inbanel & Incidence of short-term bank debt & $\begin{array}{l}\text { Ratio of short-term bank borrowings and shareholders' } \\
\text { equity }\end{array}$ \\
\hline inbanol & $\begin{array}{l}\text { Incidence of medium and long-term bank } \\
\text { debt }\end{array}$ & $\begin{array}{l}\text { ratio of medium and long-term bank debt and } \\
\text { shareholders' equity }\end{array}$ \\
\hline inalen & $\begin{array}{l}\text { Incidence of debts to other financiers in } \\
\text { the short term }\end{array}$ & $\begin{array}{l}\text { Ratio of debt to other financiers in the short term and } \\
\text { shareholders' equity }\end{array}$ \\
\hline inalol & $\begin{array}{l}\text { Incidence of debts to other financiers in } \\
\text { the medium to long term }\end{array}$ & $\begin{array}{l}\text { Ratio of debt to other financiers in the medium and } \\
\text { long-term and shareholders' equity }\end{array}$ \\
\hline curr & Current ratio & Ratio of current assets and current liabilities \\
\hline solven & Solvency ratio & Ratio of equity to total assets \\
\hline cosden & Impact of financial charges & $\begin{array}{l}\text { The ratio of total financial charges and the sum of short } \\
\text { and medium - long-term bank debt }\end{array}$ \\
\hline Idim & Firm's size & Natural logarithm of the number of employees \\
\hline logeta & Firm's age & $\begin{array}{l}\text { Natural logarithm of firm's age at the time of the } \\
\text { analysis }\end{array}$ \\
\hline darea & Dummy variable for the territoriality & $\begin{array}{l}\text { Dummy equal to } 1 \text { if the firm is located in the Centre } \\
\text { and North of Italy }\end{array}$ \\
\hline
\end{tabular}

Where $i$ identifies the single observations of the sample; $Y_{i k}$ is the dependent variable expressed by $k$ independent variables; $b_{1}, b_{2}$,... are the parameters that have to be estimated with the model; $\alpha$ is the constant and $\varepsilon$ is the error. Enterprises may adopt a combination of trade receivables and payables that is coherent both in terms of amount as in terms of duration. To verify the first research hypothesis this analysis considers two regression models in which the dependent variables are: the incidence of account receivables on total assets ( $\mathrm{cr} / \mathrm{ta}$ ) and the incidence of account payables on total assets (deforta). The difference between the two models consists in the presence (net models) or not (gross models) of an extra independent variable (varspec), specular in relation to the dependent variable. In the net model that shows as the dependent variable the incidence of trade receivables on total assets, the incidence of trade payables is considered among the explanatory variables. In the net model that shows as the dependent variable the incidence of trade payables on total assets, the incidence of trade receivables is considered among the explanatory variables (Fabbri and Klapper 2008, Gibilaro and Mattarocci 2010). If the decision to include the specular variable results statistically significant and determines an improvement in the statistical significance of the model, trade credit policy and trade debt policy can be considered closely interrelated. 
To verify the second and third research hypotheses this analysis considers only the results of the gross models. Useful predictors are inbanen, inbanol, inalen, inalol, cosden, related to the incidence of debt towards banks and third parties - for short and long-term periods - on shareholder equity and the rate of financial charges on the total bank debt. The independent control variables are related to the general characteristics of the firms (Idim, logeta and darea) and to the financial and economical balance conditions (ros, roe, curr, solven).

The existence of a significant dependency relationship between trade payables and receivables and the independent financial variables could confirm the use of intercompany credit for financial purposes.

The existence of a positive relationship of dependency between the dependent variables and bank loans could show system conditions which allow a complementary use of intercompany financing and bank financing. The existence of a negative relationship of dependency could signal an alternate use of intercompany credit with respect to bank financing. The dynamic of the ratio between net mercantile credit and total assets (crmerta) is also investigated. Net mercantile credit is very significant for the purpose of analysis, as it represents the amount of working capital that may be covered by financial debts. The existence of significant dependent relationships of the variable crmerta compared to the independent financial variables could confirm the importance of financial motivations in the recourse in trade credit.

The reference sample is composed of SMEs where the balance sheets were available in the Aida Bureau Van Dijk database in November 2013.

The enterprises present the following characteristics:

- Legal status: active firms; independent firms;

- Joint stock companies and limited companies;

- Number of employees less than 250;

- Total assets less than 43 million euros;

- Financial statements availability: for the years 2006 to 2011.

The study was carried out for the years 2006 - 2011 and the sample is made up of enterprises from all over Italy and very different in terms of location and business sectors, according to the classification of economic activities by the Italian National Institute of Statistics (ISTAT): ATECO classification 2007. Firms that conduct financial activities (Ateco codes: 64, 65, 66) were not included in the sample.

Table 2: Descriptive Statistics - 2006

\begin{tabular}{lrrrrr}
\hline \hline Variable & Obs & Mean & Std Dev & Min & Max \\
\hline \hline crclta & 864 & 0,370 & 0,203 & 0,000 & 0,981 \\
deforta & 864 & 0,282 & 0,162 & 0,000 & 0,909 \\
crmerta & 864 & 0,088 & 0,200 & $-0,772$ & 0,699 \\
inbanen & 864 & 1,885 & 19,831 & 0,000 & 577,306 \\
inbanol & 864 & 0,428 & 1,346 & 0,000 & 27,590 \\
inalen & 864 & 0,046 & 0,458 & 0,000 & 11,746 \\
inalol & 864 & 0,027 & 0,178 & 0,000 & 2,215 \\
cosden & 722 & 5,693 & 3,974 & 0,000 & 19,750 \\
ros & 843 & 5,462 & 6,647 & $-42,490$ & 29,110 \\
roe & 861 & 8,337 & 16,403 & $-109,620$ & 88,490 \\
curr & 863 & 1,647 & 0,969 & 0,020 & 9,650 \\
solven & 865 & 0,308 & 0,197 & 0,001 & 0,994 \\
Idim & 865 & 9,041 & 0,806 & 6,032 & 10,544 \\
logeta & 865 & 3,192 & 0,593 & 1,099 & 4,595 \\
darea & 865 & 0,894 & 0,308 & 0,000 & 1,000 \\
\hline \hline \multicolumn{6}{c}{}
\end{tabular}


Table 3: Descriptive statistics - 2011

\begin{tabular}{lrrrrr}
\hline \hline Variable & Obs & Mean & Std Dev & Min & Max \\
\hline \hline crclta & 865 & 0,327 & 0,200 & 0,000 & 0,966 \\
deforta & 865 & 0,230 & 0,145 & 0,000 & 0,892 \\
crmerta & 865 & 0,097 & 0,182 & $-0,860$ & 0,752 \\
inbanen & 865 & 1,607 & 19,154 & 0,000 & 559,466 \\
inbanol & 865 & 0,397 & 1,076 & 0,000 & 15,922 \\
inalen & 865 & 0,028 & 0,318 & 0,000 & 8,589 \\
inalol & 865 & 0,017 & 0,151 & 0,000 & 3,877 \\
cosden & 736 & 4,252 & 3,183 & 0,000 & 19,940 \\
ros & 839 & 3,649 & 6,869 & $-47,000$ & 29,130 \\
roe & 861 & 3,843 & 18,467 & $-130,320$ & 97,400 \\
curr & 861 & 1,745 & 1,146 & 0,120 & 9,820 \\
solven & 865 & 0,367 & 0,213 & 0,001 & 0,989 \\
Idim & 865 & 9,252 & 0,823 & 5,439 & 10,632 \\
logeta & 865 & 3,406 & 0,466 & 2,079 & 4,644 \\
darea & 865 & 0,894 & 0,308 & 0,000 & 1,000 \\
\hline \hline
\end{tabular}

In order to verify the third research hypothesis, the sample is divided into industrial and service firms: industrial firms belonging to ATECO sectors 01-53 and service firms belonging to ATECO sectors 55-99.

From a geographical point of view most of the firms are located in the North: the sample, therefore, reflects the economic and productive Italian systems, which are characterized by a greater number of enterprises located in the northern regions compared to the firms which are located in southern and central regions.

The descriptive statistics are calculated on the available data for the first and last year considered and they are presented in Tables no. 2 and 3.

\section{FINDINGS AND DISCUSSIONS}

The linear regression models are statistically significant in all years under observation, for all dependent variables considered alternatingly. The results of the collinearity tests allow us to exclude problems of collinearity: the values of the Variance Inflation Factor (VIF) are always inferior to the critical threshold of literature (Fox 1997). The results of the White heteroskedasticity test demonstrate heteroskedasticity problems. Therefore robust standard errors analyses are conducted.

The first research hypothesis is verified by comparing the gross models (Tables no. 4 and 6), that don't consider the specular variable as a regressor, and the net models (Tables no. 5 and 7), that consider the specular variable as a regressor (varspec). The comparison between gross and net models shows that the net models allow an improvement of the analysis (the value of R-squared increases significantly). We can, therefore, assume - in line with reference literature (Fabbri and Klapper 2008, Gibilaro and Mattarocci 2010) and confirming the first research hypothesis - that there is an interdependent relationship between trade credit policy and trade debt policy. 
Table 4: Regression Analysis 2006-2011 - Dependent Variable: crclta - gross models

\begin{tabular}{|c|c|c|c|c|c|c|c|c|c|c|c|c|}
\hline & 2006 & & 2007 & & 2008 & & 2009 & & 2010 & & 2011 & \\
\hline \multirow[t]{2}{*}{ inbanen } & 0,0008 & $* * *$ & 0,0008 & $* * *$ & 0,0009 & $* * *$ & 0,0011 & $* * *$ & 0,0011 & $* * *$ & 0,0009 & $*$ \\
\hline & 0,0000 & & 0,0000 & & 0,0000 & & 0,0000 & & 0,0000 & & 0,0000 & \\
\hline \multirow[t]{2}{*}{ inbanol } & $-0,0280$ & $* * *$ & $-0,0222$ & $* *$ & $-0,0549$ & $* *$ & $-0,0739$ & $* * *$ & $-0,0503$ & $* * *$ & $-0,0351$ & $* * *$ \\
\hline & 0,0009 & & 0,0325 & & 0,0170 & & 0,0000 & & 0,0051 & & 0,0009 & \\
\hline \multirow[t]{2}{*}{ inalen } & $-0,0213$ & $* *$ & 0,0080 & & $-0,0166$ & & 0,0323 & & $-0,0100$ & & 0,0217 & \\
\hline & 0,0319 & & 0,8837 & & 0,5996 & & 0,3792 & & 0,2639 & & 0,1922 & \\
\hline \multirow[t]{2}{*}{ inalol } & $-0,0412$ & & $-0,0234$ & & 0,0086 & & $-0,0027$ & & 0,0337 & & 0,0256 & \\
\hline & 0,3584 & & 0,3490 & & 0,5677 & & 0,9039 & & 0,3991 & & 0,7973 & \\
\hline \multirow[t]{2}{*}{ cosden } & $-0,0024$ & & $-0,0027$ & & 0,0006 & & $-0,0047$ & $* *$ & $-0,0078$ & $* * *$ & $-0,0075$ & $* * *$ \\
\hline & 0,1841 & & 0,1394 & & 0,7475 & & 0,0226 & & 0,0001 & & 0,0002 & \\
\hline \multirow[t]{2}{*}{ ros } & $-0,0038$ & $* *$ & $-0,0041$ & $* *$ & $-0,0028$ & $*$ & $-0,0004$ & & $-0,0004$ & & $-0,0027$ & $*$ \\
\hline & 0,0386 & & 0,0139 & & 0,0560 & & 0,7700 & & 0,7694 & & 0,0864 & \\
\hline \multirow[t]{2}{*}{ roe } & 0,0016 & $* * *$ & 0,0020 & $* * *$ & 0,0032 & $* * *$ & 0,0019 & $* * *$ & 0,0018 & $* * *$ & 0,0023 & $* * *$ \\
\hline & 0,0012 & & 0,0003 & & 0,0000 & & 0,0006 & & 0,0092 & & 0,0000 & \\
\hline \multirow[t]{2}{*}{ curr } & 0,0419 & $* * *$ & 0,0411 & $* * *$ & 0,0393 & $* * *$ & 0,0372 & $* * *$ & 0,0466 & $* * *$ & 0,0447 & $* * *$ \\
\hline & 0,0046 & & 0,0044 & & 0,0001 & & 0,0000 & & 0,0001 & & 0,0002 & \\
\hline \multirow[t]{2}{*}{ solven } & $-0,3650$ & $* * *$ & $-0,3453$ & $* * *$ & $-0,4933$ & $* * *$ & $-0,5470$ & $* * *$ & $-0,5350$ & $* * *$ & $-0,5463$ & $* * *$ \\
\hline & 0,0000 & & 0,0000 & & 0,0000 & & 0,0000 & & 0,0000 & & 0,0000 & \\
\hline \multirow[t]{2}{*}{ Idim } & $-0,0074$ & & $-0,0012$ & & $-0,0101$ & & $-0,0275$ & $* * *$ & $-0,0192$ & $*$ & $-0,0190$ & $* *$ \\
\hline & 0,4528 & & 0,8957 & & 0,2619 & & 0,0059 & & 0,0589 & & 0,0496 & \\
\hline \multirow[t]{2}{*}{ logeta } & 0,0091 & & 0,0115 & & 0,0037 & & $-0,0044$ & & $-0,0072$ & & $-0,0007$ & \\
\hline & 0,4285 & & 0,3426 & & 0,7410 & & 0,7224 & & 0,5732 & & 0,9595 & \\
\hline \multirow[t]{2}{*}{ darea } & 0,1126 & $* * *$ & 0,1159 & $* * *$ & 0,0700 & $* * *$ & 0,0655 & $* * *$ & 0,0698 & $* *$ & 0,0417 & \\
\hline & 0,0000 & & 0,0000 & & 0,0043 & & 0,0063 & & 0,0103 & & 0,1313 & \\
\hline
\end{tabular}

deforta

crclta

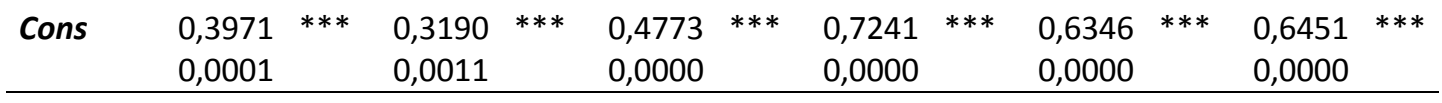

\begin{tabular}{lrrrrrr}
$N$ & 707 & 711 & 702 & 707 & 704 & 720 \\
$\mathrm{R}^{2}$ & 0,1497 & 0,1635 & 0,2395 & 0,2659 & 0,2529 & 0,2704 \\
$\mathrm{~F}$ & 16,3329 & 27,4496 & 23,2727 & 26,8994 & 22,4732 & 26,3636 \\
\hline
\end{tabular}

Level of significance: $\left({ }^{* *}\right) 1 \% ;\left(^{* *}\right) 5 \% ;(*) 10 \%$ 
Table 5: Regression Analysis 2006-2011 - Dependent Variable: crclta - net models

\begin{tabular}{|c|c|c|c|c|c|c|c|c|c|c|c|c|}
\hline & 2006 & & 2007 & & 2008 & & 2009 & & 2010 & & 2011 & \\
\hline \multirow[t]{2}{*}{ inbanen } & 0,0009 & $* * *$ & 0,0009 & $* * *$ & 0,0010 & $* * *$ & 0,0012 & $* * *$ & 0,0012 & $* * *$ & 0,0011 & $* * *$ \\
\hline & 0,0000 & & 0,0000 & & 0,0000 & & 0,0000 & & 0,0000 & & 0,0000 & \\
\hline \multirow[t]{2}{*}{ inbanol } & $-0,0193$ & $* * *$ & $-0,0162$ & $*$ & $-0,0404$ & $* *$ & $-0,0568$ & $* * *$ & $-0,0339$ & $* * *$ & $-0,0257$ & $* * *$ \\
\hline & 0,0038 & & 0,0521 & & 0,0156 & & 0,0000 & & 0,0086 & & 0,0007 & \\
\hline \multirow[t]{2}{*}{ inalen } & $-0,0317$ & $* * *$ & $-0,0014$ & & $-0,0041$ & & 0,0451 & $*$ & $-0,0001$ & & 0,0318 & $* *$ \\
\hline & 0,0010 & & 0,9814 & & 0,8658 & & 0,0969 & & 0,9931 & & 0,0308 & \\
\hline \multirow[t]{2}{*}{ inalol } & $-0,0202$ & & $-0,0100$ & & 0,0018 & & $-0,0039$ & & 0,0547 & & 0,0737 & \\
\hline & 0,6192 & & 0,6734 & & 0,8982 & & 0,8275 & & 0,1482 & & 0,4379 & \\
\hline \multirow[t]{2}{*}{ cosden } & $-0,0041$ & $* *$ & $-0,0038$ & $* *$ & 0,0008 & & $-0,0057$ & $* * *$ & $-0,0078$ & $* * *$ & $-0,0078$ & $* * *$ \\
\hline & 0,0295 & & 0,0415 & & 0,6695 & & 0,0057 & & 0,0001 & & 0,0001 & \\
\hline \multirow[t]{2}{*}{ ros } & $-0,0007$ & & $-0,0010$ & & $-0,0009$ & & 0,0003 & & 0,0002 & & $-0,0015$ & \\
\hline & 0,6413 & & 0,4595 & & 0,4361 & & 0,7985 & & 0,8796 & & 0,2287 & \\
\hline \multirow[t]{2}{*}{ roe } & 0,0007 & & 0,0011 & $* *$ & 0,0021 & $* * *$ & 0,0012 & $* *$ & 0,0009 & & 0,0017 & $* * *$ \\
\hline & 0,1728 & & 0,0418 & & 0,0001 & & 0,0217 & & 0,1674 & & 0,0009 & \\
\hline \multirow[t]{2}{*}{ curr } & 0,0475 & $* * *$ & 0,0456 & $* * *$ & 0,0387 & $* * *$ & 0,0349 & $* * *$ & 0,0467 & $* * *$ & 0,0406 & $* * *$ \\
\hline & 0,0003 & & 0,0003 & & 0,0000 & & 0,0000 & & 0,0000 & & 0,0001 & \\
\hline \multirow[t]{2}{*}{ solven } & $-0,1952$ & $* * *$ & $-0,1747$ & $* * *$ & $-0,2833$ & $* * *$ & $-0,3373$ & $* * *$ & $-0,2978$ & $* * *$ & $-0,3134$ & $* * *$ \\
\hline & 0,0010 & & 0,0046 & & 0,0000 & & 0,0000 & & 0,0000 & & 0,0000 & \\
\hline \multirow[t]{2}{*}{ Idim } & $-0,0002$ & & 0,0075 & & $-0,0023$ & & $-0,0159$ & & $-0,0108$ & & $-0,0125$ & \\
\hline & 0,9859 & & 0,3806 & & 0,7875 & & 0,1042 & & 0,2601 & & 0,1686 & \\
\hline \multirow[t]{2}{*}{ logeta } & 0,0042 & & 0,0053 & & $-0,0022$ & & $-0,0096$ & & $-0,0106$ & & $-0,0056$ & \\
\hline & 0,7018 & & 0,6493 & & 0,8411 & & 0,3991 & & 0,3560 & & 0,6359 & \\
\hline \multirow[t]{2}{*}{ darea } & 0,1052 & $* * *$ & 0,1115 & $* * *$ & 0,0807 & $* * *$ & 0,0754 & $* * *$ & 0,0766 & $* * *$ & 0,0556 & $* *$ \\
\hline & 0,0000 & & 0,0000 & & 0,0012 & & 0,0016 & & 0,0036 & & 0,0315 & \\
\hline \multirow[t]{2}{*}{ deforta } & 0,4670 & $* * *$ & 0,5023 & $* * *$ & 0,4972 & $* * *$ & 0,4722 & $* * *$ & 0,5369 & $* * *$ & 0,5254 & $* * *$ \\
\hline & 0,0000 & & 0,0000 & & 0,0000 & & 0,0000 & & 0,0000 & & 0,0000 & \\
\hline \multicolumn{13}{|l|}{ crclta } \\
\hline \multirow[t]{2}{*}{ Cons } & 0,1592 & & 0,0631 & & 0,2146 & $* *$ & 0,4449 & $* * *$ & 0,3466 & $* * *$ & 0,3846 & $* * *$ \\
\hline & 0,1104 & & 0,4978 & & 0,0236 & & 0,0000 & & 0,0013 & & 0,0003 & \\
\hline $\mathrm{N}$ & 707 & & 711 & & 702 & & 707 & & 704 & & 720 & \\
\hline$R^{2}$ & 0,2394 & & 0,2656 & & 0,3254 & & 0,3377 & & 0,3529 & & 0,3659 & \\
\hline $\mathrm{F}$ & 21,1921 & & 27,1594 & & 27,8388 & & 33,3822 & & 34,1973 & & 39,6877 & \\
\hline
\end{tabular}

Level of significance: $\left({ }^{* * *}\right) 1 \% ;\left({ }^{* *}\right) 5 \% ;\left({ }^{*}\right) 10 \%$

The verification of the second research hypothesis takes into account the results of the gross models (Tables no. 4 and 6) and is related to the coexistence of substitutability and complementarity conditions between trade credit and debts with third parties or banks. The importance of financial motivations is evident in the relationship between the dependent variables crclta, deforta and crmerta and the incidence of debt towards banks and third parties, both for short and medium and long term. 
Table 6: Regression Analysis 2006-2011 - Dependent Variable: deforta - gross models

\begin{tabular}{|c|c|c|c|c|c|c|c|c|c|c|c|c|}
\hline & 2006 & & 2007 & & 2008 & & 2009 & & 2010 & & 2011 & \\
\hline \multirow[t]{2}{*}{ inbanen } & $-0,0002$ & $* * *$ & $-0,0002$ & $* * *$ & $-0,0003$ & $* * *$ & $-0,0003$ & $* * *$ & $-0,0002$ & $* * *$ & $-0,0003$ & $* * *$ \\
\hline & 0,0000 & & 0,0005 & & 0,0000 & & 0,0000 & & 0,0002 & & 0,0000 & \\
\hline \multirow[t]{2}{*}{ inbanol } & $-0,0188$ & $* * *$ & $-0,0120$ & $* *$ & $-0,0291$ & $*$ & $-0,0364$ & $* * *$ & $-0,0305$ & $* * *$ & $-0,0180$ & $* *$ \\
\hline & 0,0008 & & 0,0138 & & 0,0521 & & 0,0010 & & 0,0094 & & 0,0209 & \\
\hline \multirow[t]{2}{*}{ inalen } & 0,0223 & $* * *$ & 0,0188 & & $-0,0253$ & & $-0,0270$ & & $-0,0184$ & $* * *$ & $-0,0193$ & $* * *$ \\
\hline & 0,0000 & & 0,6722 & & 0,1612 & & 0,4384 & & 0,0000 & & 0,0033 & \\
\hline \multirow[t]{2}{*}{ inalol } & $-0,0451$ & $* *$ & $-0,0266$ & & 0,0137 & & 0,0025 & & $-0,0390$ & & $-0,0915$ & $* *$ \\
\hline & 0,0406 & & 0,1136 & & 0,1282 & & 0,8780 & & 0,1882 & & 0,0133 & \\
\hline \multirow[t]{2}{*}{ cosden } & 0,0035 & $* * *$ & 0,0021 & $*$ & $-0,0004$ & & 0,0021 & & 0,0000 & & 0,0007 & \\
\hline & 0,0087 & & 0,0984 & & 0,7663 & & 0,1200 & & 0,9899 & & 0,6685 & \\
\hline \multirow[t]{2}{*}{ ros } & $-0,0066$ & $* * *$ & $-0,0061$ & $* * *$ & $-0,0038$ & $* * *$ & $-0,0013$ & & $-0,0011$ & & $-0,0024$ & $* *$ \\
\hline & 0,0000 & & 0,0000 & & 0,0012 & & 0,1332 & & 0,2951 & & 0,0158 & \\
\hline \multirow[t]{2}{*}{ roe } & 0,0021 & $* * *$ & 0,0019 & $* * *$ & 0,0021 & $* * *$ & 0,0014 & $* * *$ & 0,0016 & $* * *$ & 0,0012 & $* * *$ \\
\hline & 0,0000 & & 0,0000 & & 0,0001 & & 0,0047 & & 0,0005 & & 0,0047 & \\
\hline \multirow[t]{2}{*}{ curr } & $-0,0121$ & & $-0,0089$ & & 0,0012 & & 0,0046 & & $-0,0002$ & & 0,0078 & \\
\hline & 0,1441 & & 0,2422 & & 0,8237 & & 0,2972 & & 0,9767 & & 0,1704 & \\
\hline \multirow[t]{2}{*}{ solven } & $-0,3634$ & $* * *$ & $-0,3398$ & $* * *$ & $-0,4225$ & $* * *$ & $-0,4444$ & $* * *$ & $-0,4419$ & $* * *$ & $-0,4432$ & $* * *$ \\
\hline & 0,0000 & & 0,0000 & & 0,0000 & & 0,0000 & & 0,0000 & & 0,0000 & \\
\hline \multirow[t]{2}{*}{ Idim } & $-0,0154$ & $* *$ & $-0,0173$ & $* * *$ & $-0,0158$ & $* * *$ & $-0,0242$ & $* * *$ & $-0,0154$ & $* *$ & $-0,0123$ & $* *$ \\
\hline & 0,0123 & & 0,0045 & & 0,0094 & & 0,0000 & & 0,0167 & & 0,0447 & \\
\hline \multirow[t]{2}{*}{ logeta } & 0,0105 & & 0,0124 & & 0,0119 & & 0,0109 & & 0,0062 & & 0,0095 & \\
\hline & 0,2213 & & 0,1694 & & 0,1665 & & 0,2081 & & 0,5049 & & 0,3137 & \\
\hline \multirow[t]{2}{*}{ darea } & 0,0158 & & 0,0089 & & $-0,0215$ & & $-0,0212$ & & $-0,0127$ & & $-0,0264$ & \\
\hline & 0,3873 & & 0,6635 & & 0,2397 & & 0,2147 & & 0,5043 & & 0,1794 & \\
\hline \multicolumn{13}{|l|}{ deforta } \\
\hline \multicolumn{13}{|l|}{ crclta } \\
\hline \multirow[t]{2}{*}{ Cons } & 0,5095 & $* * *$ & 0,5095 & $* * *$ & 0,5284 & $* * *$ & 0,5886 & $* * *$ & 0,5357 & $* * *$ & 0,4959 & $* * *$ \\
\hline & 0,0000 & & 0,0000 & & 0,0000 & & 0,0000 & & 0,0000 & & 0,0000 & \\
\hline $\mathrm{N}$ & 707 & & 711 & & 702 & & 708 & & 705 & & 720 & \\
\hline $\mathrm{R}^{2}$ & 0,3212 & & 0,2927 & & 0,3416 & & 0,3583 & & 0,3325 & & 0,3174 & \\
\hline $\mathrm{F}$ & 29,4879 & & 24,0806 & & 26,7834 & & 34,1884 & & 35,4781 & & 26,1487 & \\
\hline
\end{tabular}

Level of significance: $\left({ }^{* * *}\right) 1 \%$; $\left({ }^{* *}\right) 5 \%$; $\left({ }^{*}\right) 10 \%$ 
Table 7: Regression Analysis 2006-2011 - Dependent Variable: deforta - net models

\begin{tabular}{|c|c|c|c|c|c|c|c|c|c|c|c|c|}
\hline & 2006 & & 2007 & & 2008 & & 2009 & & 2010 & & 2011 & \\
\hline \multirow[t]{2}{*}{ inbanen } & $-0,0004$ & $* * *$ & $-0,0004$ & $* * *$ & $-0,0005$ & $* * *$ & $-0,0005$ & $* * *$ & $-0,0005$ & $* * *$ & $-0,0005$ & $* * *$ \\
\hline & 0,0000 & & 0,0000 & & 0,0000 & & 0,0000 & & 0,0000 & & 0,0000 & \\
\hline \multirow[t]{2}{*}{ inbanol } & $-0,0125$ & $* * *$ & $-0,0066$ & $* *$ & $-0,0167$ & & $-0,0211$ & $* *$ & $-0,0179$ & $* *$ & $-0,0092$ & \\
\hline & 0,0042 & & 0,0225 & & 0,1253 & & 0,0438 & & 0,0301 & & 0,1227 & \\
\hline \multirow[t]{2}{*}{ inalen } & 0,0271 & $* * *$ & 0,0168 & & $-0,0215$ & $*$ & $-0,0337$ & & $-0,0159$ & $* * *$ & $-0,0247$ & $* * *$ \\
\hline & 0,0000 & & 0,7188 & & 0,0722 & & 0,2565 & & 0,0000 & & 0,0000 & \\
\hline \multirow[t]{2}{*}{ inalol } & $-0,0357$ & $*$ & $-0,0209$ & & 0,0118 & & 0,0031 & & $-0,0474$ & $*$ & $-0,0978$ & $* * *$ \\
\hline & 0,0583 & & 0,1989 & & 0,1470 & & 0,8170 & & 0,0904 & & 0,0079 & \\
\hline \multirow[t]{2}{*}{ cosden } & 0,0041 & $* * *$ & 0,0028 & $* *$ & $-0,0005$ & & 0,0030 & $* *$ & 0,0020 & & 0,0025 & $*$ \\
\hline & 0,0026 & & 0,0321 & & 0,6809 & & 0,0252 & & 0,1996 & & 0,0952 & \\
\hline \multirow[t]{2}{*}{ ros } & $-0,0058$ & $* * *$ & $-0,0051$ & $* * *$ & $-0,0032$ & $* * *$ & $-0,0012$ & $*$ & $-0,0010$ & & $-0,0017$ & $* *$ \\
\hline & 0,0000 & & 0,0000 & & 0,0013 & & 0,0881 & & 0,2335 & & 0,0185 & \\
\hline \multirow[t]{2}{*}{ roe } & 0,0017 & $* * *$ & 0,0014 & $* * *$ & 0,0014 & $* * *$ & 0,0010 & $* *$ & 0,0012 & $* * *$ & 0,0006 & \\
\hline & 0,0001 & & 0,0001 & & 0,0030 & & 0,0342 & & 0,0084 & & 0,1044 & \\
\hline \multirow[t]{2}{*}{ curr } & $-0,0215$ & $* * *$ & $-0,0189$ & $* * *$ & $-0,0077$ & $*$ & $-0,0028$ & & $-0,0118$ & $* * *$ & $-0,0034$ & \\
\hline & 0,0028 & & 0,0023 & & 0,0824 & & 0,4607 & & 0,0086 & & 0,4529 & \\
\hline \multirow[t]{2}{*}{ solven } & $-0,2810$ & $* * *$ & $-0,2559$ & $* * *$ & $-0,3105$ & $* * *$ & $-0,3308$ & $* * *$ & $-0,3084$ & $* * *$ & $-0,3070$ & $* * *$ \\
\hline & 0,0000 & & 0,0000 & & 0,0000 & & 0,0000 & & 0,0000 & & 0,0000 & \\
\hline \multirow[t]{2}{*}{ Idim } & $-0,0138$ & $* *$ & $-0,0170$ & $* * *$ & $-0,0135$ & $* *$ & $-0,0189$ & $* * *$ & $-0,0107$ & $*$ & $-0,0076$ & \\
\hline & 0,0171 & & 0,0028 & & 0,0184 & & 0,0009 & & 0,0853 & & 0,1825 & \\
\hline \multirow[t]{2}{*}{ logeta } & 0,0084 & & 0,0096 & & 0,0110 & & 0,0120 & & 0,0081 & & 0,0096 & \\
\hline & 0,3011 & & 0,2613 & & 0,1768 & & 0,1357 & & 0,3385 & & 0,2613 & \\
\hline \multirow[t]{2}{*}{ darea } & $-0,0097$ & & $-0,0193$ & & $-0,0374$ & $* *$ & $-0,0347$ & $* *$ & $-0,0301$ & $*$ & $-0,0368$ & $* *$ \\
\hline & 0,5839 & & 0,3103 & & 0,0408 & & 0,0393 & & 0,0955 & & 0,0460 & \\
\hline \multicolumn{13}{|l|}{ deforta } \\
\hline \multirow[t]{2}{*}{ crclta } & 0,2259 & $* * *$ & 0,2431 & $* * *$ & 0,2270 & $* * *$ & 0,2071 & $* * *$ & 0,2493 & $* * *$ & 0,2492 & $* * *$ \\
\hline & 0,0000 & & 0,0000 & & 0,0000 & & 0,0000 & & 0,0000 & & 0,0000 & \\
\hline \multirow[t]{2}{*}{ Cons } & 0,4198 & $* * *$ & 0,4319 & $* * *$ & 0,4200 & $* * *$ & 0,4413 & $* * *$ & 0,3783 & $* * *$ & 0,3351 & $* * *$ \\
\hline & 0,0000 & & 0,0000 & & 0,0000 & & 0,0000 & & 0,0000 & & 0,0000 & \\
\hline $\mathrm{N}$ & 707 & & 711 & & 702 & & 707 & & 704 & & 720 & \\
\hline$R^{2}$ & 0,3928 & & 0,3791 & & 0,4159 & & 0,4206 & & 0,4217 & & 0,4068 & \\
\hline $\mathrm{F}$ & 37,1387 & & 32,9192 & & 36,6864 & & 38,3653 & & 43,9904 & & 36,4651 & \\
\hline
\end{tabular}

Level of significance: $\left({ }^{* * *}\right) 1 \% ;\left({ }^{* *}\right) 5 \% ;\left({ }^{*}\right) 10 \%$ 
There is a statistically significant relationship between all dependent variables and the variables which show the incidence of debt towards banks for a short term (inbanel) and a medium and long term (inbanol). In the six years, it is possible to observe a statistically significant negative relationship between the incidence of trade payables (deforta) and the incidence of short-term bank debt (inbanen) and medium and long-term bank debt (inbanol). The presence of an inverse dependent relationship highlights substitution conditions in the use of different sources of financing: a greater recourse in bank financing results in a lower recourse in trade credits.

Regarding the incidence of trade receivables ( $\mathrm{crclta}$ ) instead, the relationship is positive and direct with the incidence of short-term bank debt (inbanel) and negative with the incidence of medium and long-term bank debt (inbanol). The presence of a significant positive dependent relationship between the incidence of trade credits and the incidence of bank loans or third parties is a symptom of double intermediary conditions, therefore of complementarity between credit from third parties and trade credit. The presence of a direct relationship between the variable crclta and the short-term bank financing states that there are conditions of complementarity and emphasizes the importance of transactional motivations other than solely financial in the granting of trade credit. The coexistence of complementarity and substitutability conditions between intercompany credit and financing from banks or third parties is also confirmed by the results of the regression model (Table no. 8) which considers the dependent variable crmerta (incidence of mercantile credit on total assets). The existence of a significant and positive dependent relationship between crmerta and external financing sources sustains the hypothesis of complementary use of trade credit compared to financing from banks: enterprises try to minimize the amount of financial needs in situations of credit restrictions and increase it in times of greater availability of bank financing. Conversely, a negative dependent relationship highlights an alternative use of trade credit compared to financing from banks, showing a minimization of financial needs in situations of credit expansion.

The empirical analysis confirms the coexistence of complementarity and substitutability conditions, as the crmerta and crclta variables present a significant positive relationship with short-term bank financing (inbanel) and a significant and negative relationship with medium and long-term bank financing (inbanol), in the six years. It is interesting to consider the relationship between the dependent variables and the regressor cosden (the ratio of financial charges on total bank debt): the relationship is not consistently significant for every year studied. The relationship is statistically significant and positive in the years 2006 and 2007 between deforta and cosden (Table no. 6): when the cost of borrowing increases the incidence of trade payables also increases, showing conditions of substitutability between bank loans and inter-company loans. The relationship is statistically significant and negative in the years 2009-2011 between crclta and cosden (Table no. 4): during crisis years, if the cost of money increases the cost of borrowing increases and double intermediary conditions may be too expensive, so credits granted to customers decrease. It is also possible to observe statistically significant relationships between the deforta variable with other predictive finance variables (Table no. 6). In particular, we can observe a significant and negative relationship with the inalen and inalo/ variables, but not in all years under observation. The relationship with the predictor variable inalen is statistically significant in 2006 and 2010 -2011; the relationship with the incidence of medium and long-term loans from third parties (inanol) is statistically significant only in 2006 and 2011. The presence of a negative dependent relationship in the years 2010 and 2011 highlights substitution conditions: a greater recourse in third party financing results in a lower recourse in trade credits. The medium and long-term financial predictive variables show a greater importance following an increase in the financial and credit crisis. Regarding firm's characteristics, we can see the impact of the darea variable (dummy which has a value 1 for firms located in the central and northern areas of Italy and 0 for firms located in the southern regions and islands) on crclta and crmerta (Tables no. 4 and 8). 
Table 8: Regression Analysis 2006-2011 - Dependent Variable: crmerta

\begin{tabular}{|c|c|c|c|c|c|c|c|c|c|c|c|c|}
\hline & 2006 & & 2007 & & 2008 & & 2009 & & 2010 & & 2011 & \\
\hline \multirow[t]{2}{*}{ inbanen } & 0,0010 & $* * *$ & 0,0010 & $* * *$ & 0,0012 & $* * *$ & 0,0013 & $* * *$ & 0,0013 & $* * *$ & 0,0012 & $* * *$ \\
\hline & 0,0000 & & 0,0000 & & 0,0000 & & 0,0000 & & 0,0000 & & 0,0000 & \\
\hline \multirow[t]{2}{*}{ inbanol } & $-0,0092$ & $*$ & $-0,0102$ & & $-0,0258$ & $* *$ & $-0,0376$ & $* * *$ & $-0,0198$ & $* *$ & $-0,0171$ & $* * *$ \\
\hline & 0,0753 & & 0,1064 & & 0,0136 & & 0,0024 & & 0,0190 & & 0,0020 & \\
\hline \multirow[t]{2}{*}{ inalen } & $-0,0436$ & $* * *$ & $-0,0107$ & & 0,0087 & & 0,0593 & $* *$ & 0,0085 & & 0,0410 & $* * *$ \\
\hline & 0,0000 & & 0,8807 & & 0,6102 & & 0,0234 & & 0,2140 & & 0,0022 & \\
\hline \multirow[t]{2}{*}{ inalol } & 0,0038 & & 0,0032 & & $-0,0051$ & & $-0,0052$ & & 0,0727 & $*$ & 0,1171 & \\
\hline & 0,9206 & & 0,9014 & & 0,7071 & & 0,7438 & & 0,0772 & & 0,2113 & \\
\hline \multirow[t]{2}{*}{ cosden } & $-0,0060$ & $* * *$ & $-0,0048$ & $* *$ & 0,0010 & & $-0,0068$ & $* * *$ & $-0,0078$ & $* * *$ & $-0,0081$ & $* * *$ \\
\hline & 0,0047 & & 0,0189 & & 0,6302 & & 0,0030 & & 0,0007 & & 0,0002 & \\
\hline \multirow[t]{2}{*}{ ros } & 0,0028 & $* *$ & 0,0020 & & 0,0010 & & 0,0010 & & 0,0007 & & $-0,0004$ & \\
\hline & 0,0463 & & 0,1233 & & 0,3577 & & 0,3065 & & 0,4969 & & 0,7178 & \\
\hline \multirow[t]{2}{*}{ roe } & $-0,0004$ & & 0,0001 & & 0,0011 & $*$ & 0,0005 & & 0,0002 & & 0,0012 & $* *$ \\
\hline & 0,4338 & & 0,8173 & & 0,0556 & & 0,4201 & & 0,7970 & & 0,0225 & \\
\hline \multirow[t]{2}{*}{ curr } & 0,0539 & $* * *$ & 0,0500 & $* * *$ & 0,0381 & $* * *$ & 0,0323 & $* * *$ & 0,0468 & $* * *$ & 0,0370 & $* * *$ \\
\hline & 0,0000 & & 0,0000 & & 0,0000 & & 0,0000 & & 0,0000 & & 0,0001 & \\
\hline \multirow[t]{2}{*}{ solven } & $-0,0015$ & & $-0,0055$ & & $-0,0708$ & & $-0,1029$ & $* *$ & $-0,0932$ & $* *$ & $-0,1031$ & $* *$ \\
\hline & 0,9777 & & 0,9210 & & 0,1255 & & 0,0281 & & 0,0428 & & 0,0220 & \\
\hline \multirow[t]{2}{*}{ Idim } & 0,0081 & & 0,0161 & * & 0,0057 & & $-0,0029$ & & $-0,0037$ & & $-0,0066$ & \\
\hline & 0,3970 & & 0,0738 & & 0,5276 & & 0,7752 & & 0,7201 & & 0,4799 & \\
\hline \multirow[t]{2}{*}{ logeta } & $-0,0014$ & & $-0,0009$ & & $-0,0082$ & & $-0,0154$ & & $-0,0135$ & & $-0,0101$ & \\
\hline & 0,9060 & & 0,9444 & & 0,4938 & & 0,1989 & & 0,2642 & & 0,4206 & \\
\hline \multirow[t]{2}{*}{ darea } & 0,0968 & $* * *$ & 0,1070 & $* * *$ & 0,0915 & $* * *$ & 0,0866 & $* * *$ & 0,0825 & $* * *$ & 0,0681 & $* *$ \\
\hline & 0,0005 & & 0,0000 & & 0,0016 & & 0,0015 & & 0,0044 & & 0,0163 & \\
\hline
\end{tabular}

deforta

crclta

\begin{tabular}{lrrrrrr} 
Cons & $-0,1124$ & $-0,1905 *$ & $-0,0511$ & 0,1328 & 0,0981 & 0,1492 \\
& 0,2758 & 0,0567 & 0,6022 & 0,2050 & 0,3777 & 0,1599 \\
\hline $\mathrm{N}$ & 707 & 711 & 702 & 707 & 704 & 720 \\
$\mathrm{R}^{2}$ & 0,1255 & 0,1185 & 0,1013 & 0,1121 & 0,1164 & 0,1297 \\
$\mathrm{~F}$ & 15,1912 & 13,7945 & 10,9150 & 11,8667 & 95,4559 & 15,6929 \\
\hline
\end{tabular}

Level of significance: $\left({ }^{* *}\right) 1 \% ;\left({ }^{* *}\right) 5 \% ;\left({ }^{*}\right) 10 \%$

In all years under observation the relationship is positive and highlights the greater intensity of credit offers by firms in the central and northern regions of Italy. For the dependent variable crclta (Table no. 4) there are also statistically significant negative relationships with the predictor $/ d i m$, which represent the size of the firms, but only in 2009-2011: during the crisis years the smaller-sized firms grant extended payment deferrals, probably with the aim to strengthen client relationships. For the dependent variable deforta (Table no. 6), there are statistically significant negative relationships only with the predictor Idim, in all years under observation. The negative relationship between Idim and the incidence of trade payables shows a greater recourse in trade payables for smaller firms, confirming the hypotheses supported in literature, that trade payables are a substitute for credit from banks or third parties for small and opaque firms. To verify the third research hypothesis we replicate the analysis considering separately firms that belong to the industry sector (Tables no. 9-11) and firms that perform services (Tables no. 12-14). The linear regression models are statistically 
significant in the six years of study for all dependent variables crclta, deforta and cremerta, considered alternatingly. The results of the collinearity tests allow to exclude problems of collinearity: the values of the Variance Inflation Factor (VIF) are always inferior to the critical threshold of literature (Fox 1997). The results of the White heteroskedasticity test demonstrate heteroskedasticity problems. Therefore robust standard errors analyses are conducted.

Table 9: Regression Analysis 2006-2011 - Dependent Variable: crclta - Industry

\begin{tabular}{|c|c|c|c|c|c|c|c|c|c|c|c|c|}
\hline & 2006 & & 2007 & & 2008 & & 2009 & & 2010 & & 2011 & \\
\hline \multirow[t]{2}{*}{ inbanen } & 0,0059 & & 0,0038 & & 0,0192 & $* * *$ & 0,0327 & $* * *$ & 0,0243 & $* * *$ & 0,0143 & * \\
\hline & 0,1927 & & 0,3956 & & 0,0036 & & 0,0000 & & 0,0001 & & 0,0836 & \\
\hline \multirow[t]{2}{*}{ inbanol } & $-0,0541$ & $* * *$ & $-0,0692$ & $* * *$ & $-0,0535$ & $* * *$ & $-0,0911$ & $* * *$ & $-0,0653$ & $* * *$ & $-0,0300$ & $* * *$ \\
\hline & 0,0000 & & 0,0000 & & 0,0001 & & 0,0000 & & 0,0007 & & 0,0074 & \\
\hline \multirow[t]{2}{*}{ inalen } & 0,2152 & $* * *$ & 0,1268 & $* * *$ & $-0,0062$ & & 0,0525 & & $-0,0049$ & & 0,0075 & \\
\hline & 0,0023 & & 0,0000 & & 0,8485 & & 0,1404 & & 0,5781 & & 0,7451 & \\
\hline \multirow[t]{2}{*}{ inalol } & $-0,0861$ & $* *$ & $-0,0562$ & $* * *$ & $-0,0168$ & & $-0,0257$ & & 0,0449 & & 0,0515 & \\
\hline & 0,0275 & & 0,0060 & & 0,6114 & & 0,1735 & & 0,3835 & & 0,6664 & \\
\hline \multirow[t]{2}{*}{ cosden } & $-0,0024$ & & $-0,0012$ & & 0,0003 & & $-0,0038$ & $*$ & $-0,0064$ & $* * *$ & $-0,0048$ & $*$ \\
\hline & 0,2287 & & 0,5604 & & 0,9072 & & 0,0738 & & 0,0043 & & 0,0637 & \\
\hline \multirow[t]{2}{*}{ ros } & $-0,0037$ & $* *$ & $-0,0015$ & & $-0,0013$ & & 0,0005 & & 0,0013 & & $-0,0012$ & \\
\hline & 0,0493 & & 0,3579 & & 0,4799 & & 0,6991 & & 0,3315 & & 0,5278 & \\
\hline \multirow[t]{2}{*}{ roe } & 0,0016 & $* * *$ & 0,0012 & $* *$ & 0,0027 & $* * *$ & 0,0015 & $* *$ & 0,0012 & & 0,0022 & $* * *$ \\
\hline & 0,0029 & & 0,0287 & & 0,0008 & & 0,0325 & & 0,1032 & & 0,0003 & \\
\hline \multirow[t]{2}{*}{ curr } & 0,0381 & $* * *$ & 0,0139 & & 0,0117 & & 0,0153 & $* *$ & 0,0131 & & 0,0072 & \\
\hline & 0,0052 & & 0,3048 & & 0,1586 & & 0,0379 & & 0,1759 & & 0,5091 & \\
\hline \multirow[t]{2}{*}{ solven } & $-0,3625$ & $* * *$ & $-0,3489$ & $* * *$ & $-0,3431$ & $* * *$ & $-0,3947$ & $* * *$ & $-0,3656$ & $* * *$ & $-0,3366$ & $* * *$ \\
\hline & 0,0000 & & 0,0000 & & 0,0000 & & 0,0000 & & 0,0000 & & 0,0000 & \\
\hline \multirow[t]{2}{*}{ Idim } & $-0,0085$ & & $-0,0029$ & & $-0,0135$ & & $-0,0326$ & $* * *$ & $-0,0228$ & $*$ & $-0,0179$ & \\
\hline & 0,4842 & & 0,8023 & & 0,2600 & & 0,0099 & & 0,0711 & & 0,1736 & \\
\hline \multirow[t]{2}{*}{ logeta } & $-0,0147$ & & $-0,0242$ & $* *$ & $-0,0174$ & & $-0,0265$ & $*$ & $-0,0261$ & $*$ & $-0,0265$ & $*$ \\
\hline & 0,1988 & & 0,0444 & & 0,1678 & & 0,0522 & & 0,0629 & & 0,0813 & \\
\hline \multirow{2}{*}{ darea } & 0,1182 & $* * *$ & 0,1300 & $* * *$ & 0,0804 & $* * *$ & 0,0616 & $* *$ & 0,0857 & $* * *$ & 0,0569 & \\
\hline & \multicolumn{12}{|c|}{ deforta } \\
\hline \multicolumn{13}{|l|}{ crclta } \\
\hline \multirow[t]{2}{*}{ Cons } & 0,4839 & $* * *$ & 0,4749 & $* * *$ & 0,5407 & $* * *$ & 0,8141 & $* * *$ & 0,6889 & $* * *$ & 0,6674 & $* * *$ \\
\hline & 0,0002 & & 0,0001 & & 0,0000 & & 0,0000 & & 0,0000 & & 0,0000 & \\
\hline $\mathrm{N}$ & 455 & & 453 & & 452 & & 449 & & 455 & & 469 & \\
\hline $\mathrm{R}^{2}$ & 0,1977 & & 0,1979 & & 0,2657 & & 0,3461 & & 0,2888 & & 0,2526 & \\
\hline $\mathrm{F}$ & 9,1282 & & 8,0677 & & 11,3662 & & 20,0118 & & 17,7318 & & 11,5366 & \\
\hline
\end{tabular}

Level of significance: $\left({ }^{* * *}\right) 1 \%$; $\left({ }^{* *}\right) 5 \%$; $\left({ }^{*}\right) 10 \%$ 
Table 10: Regression Analysis 2006-2011 - Dependent Variable: defrentter-Imdtussthyy

\begin{tabular}{|c|c|c|c|c|c|c|c|c|c|c|c|c|}
\hline & 2006 & & 2007 & & 2008 & & 2009 & & 2010 & & 2011 & \\
\hline \multirow[t]{2}{*}{ inbanen } & $-0,0018$ & & $-0,0014$ & & $-0,0060$ & & $-0,0093$ & & $-0,0105$ & & $-0,0096$ & $*$ \\
\hline & 0,6059 & & 0,3237 & & 0,2361 & & 0,1800 & & 0,1277 & & 0,0870 & \\
\hline \multirow[t]{2}{*}{ inbanol } & $-0,0453$ & $* * *$ & $-0,0410$ & $* * *$ & $-0,0095$ & & $-0,0415$ & $* * *$ & $-0,0420$ & $* * *$ & $-0,0201$ & $* *$ \\
\hline & 0,0001 & & 0,0000 & & 0,4883 & & 0,0060 & & 0,0019 & & 0,0144 & \\
\hline \multirow[t]{2}{*}{ inalen } & $-0,0164$ & & 0,0039 & & $-0,0101$ & & $-0,0230$ & & $-0,0179$ & $* * *$ & $-0,0035$ & \\
\hline & 0,7724 & & 0,9292 & & 0,5386 & & 0,5811 & & 0,0000 & & 0,6979 & \\
\hline \multirow[t]{2}{*}{ inalol } & $-0,0200$ & & $-0,0090$ & & 0,0010 & & $-0,0189$ & $* *$ & $-0,0021$ & & $-0,0529$ & \\
\hline & 0,3919 & & 0,5188 & & 0,9398 & & 0,0148 & & 0,9505 & & 0,1743 & \\
\hline \multirow[t]{2}{*}{ cosden } & 0,0032 & $* *$ & 0,0025 & $*$ & 0,0002 & & 0,0017 & & $-0,0016$ & & 0,0006 & \\
\hline & 0,0341 & & 0,0870 & & 0,8699 & & 0,2397 & & 0,3700 & & 0,7590 & \\
\hline \multirow[t]{2}{*}{ ros } & $-0,0050$ & $* * *$ & $-0,0029$ & $* *$ & $-0,0018$ & & 0,0008 & & $-0,0001$ & & 0,0011 & \\
\hline & 0,0005 & & 0,0161 & & 0,1957 & & 0,3041 & & 0,9403 & & 0,2952 & \\
\hline \multirow[t]{2}{*}{ roe } & 0,0017 & $* * *$ & 0,0010 & $* *$ & 0,0016 & $* *$ & 0,0001 & & 0,0008 & & 0,0000 & \\
\hline & 0,0010 & & 0,0267 & & 0,0152 & & 0,9057 & & 0,1627 & & 0,9828 & \\
\hline \multirow[t]{2}{*}{ curr } & $-0,0208$ & $* *$ & $-0,0236$ & $* * *$ & $-0,0174$ & $* * *$ & $-0,0063$ & & $-0,0123$ & $* *$ & $-0,0096$ & \\
\hline & 0,0242 & & 0,0016 & & 0,0007 & & 0,1853 & & 0,0347 & & 0,1333 & \\
\hline \multirow[t]{2}{*}{ solven } & $-0,3670$ & $* * *$ & $-0,3193$ & $* * *$ & $-0,3159$ & $* * *$ & $-0,3966$ & $* * *$ & $-0,4152$ & $* * *$ & $-0,3700$ & $* * *$ \\
\hline & 0,0000 & & 0,0000 & & 0,0000 & & 0,0000 & & 0,0000 & & 0,0000 & \\
\hline \multirow[t]{2}{*}{ Idim } & $-0,0145$ & $*$ & $-0,0196$ & $* *$ & $-0,0138$ & $*$ & $-0,0219$ & $* * *$ & $-0,0070$ & & $-0,0066$ & \\
\hline & 0,0719 & & 0,0121 & & 0,0715 & & 0,0028 & & 0,3668 & & 0,3742 & \\
\hline \multirow[t]{2}{*}{ logeta } & 0,0030 & & 0,0062 & & 0,0111 & & 0,0061 & & 0,0002 & & 0,0053 & \\
\hline & 0,7453 & & 0,5008 & & 0,2544 & & 0,5417 & & 0,9849 & & 0,6360 & \\
\hline \multirow[t]{2}{*}{ darea } & 0,0732 & $* * *$ & 0,0689 & $* * *$ & 0,0361 & & 0,0106 & & 0,0238 & & 0,0016 & \\
\hline & 0,0026 & & 0,0078 & & 0,1587 & & 0,6573 & & 0,3911 & & 0,9587 & \\
\hline \multicolumn{13}{|l|}{ deforta } \\
\hline \multicolumn{13}{|l|}{ crclta } \\
\hline \multirow[t]{2}{*}{ Cons } & 0,4910 & $* * *$ & 0,5041 & $* * *$ & 0,4339 & $* * *$ & 0,5549 & $* * *$ & 0,4658 & $* * *$ & 0,4266 & $* * *$ \\
\hline & 0,0000 & & 0,0000 & & 0,0000 & & 0,0000 & & 0,0000 & & 0,0000 & \\
\hline $\mathrm{N}$ & 455 & & 453 & & 452 & & 450 & & 456 & & 469 & \\
\hline$R^{2}$ & 0,3344 & & 0,3175 & & 0,3184 & & 0,3311 & & 0,3303 & & 0,2755 & \\
\hline $\mathrm{F}$ & 19,3527 & & 18,5569 & & 21,3798 & & 21,7892 & & 23,0577 & & 16,8934 & \\
\hline
\end{tabular}

Level of significance: $\left({ }^{* *}\right) 1 \% ;\left({ }^{* *}\right) 5 \% ;\left({ }^{*}\right) 10 \%$ 


\begin{tabular}{|c|c|c|c|c|c|c|c|c|c|c|c|c|}
\hline \multirow{3}{*}{ inbanen } & \multicolumn{2}{|l|}{2006} & \multicolumn{2}{|l|}{2007} & \multicolumn{2}{|l|}{2008} & \multicolumn{2}{|l|}{2009} & \multicolumn{2}{|l|}{2010} & \multicolumn{2}{|l|}{2011} \\
\hline & 0,0077 & & 0,0052 & & 0,0252 & $* * *$ & 0,0420 & $* * *$ & 0,0348 & $* * *$ & 0,0239 & $* * *$ \\
\hline & 0,2962 & & 0,2864 & & 0,0000 & & 0,0000 & & 0,0000 & & 0,0026 & \\
\hline \multirow[t]{2}{*}{ inbanol } & $-0,0088$ & & $-0,0282$ & $* *$ & $-0,0440$ & $* * *$ & $-0,0496$ & $* * *$ & $-0,0233$ & $* *$ & $-0,0099$ & \\
\hline & 0,5247 & & 0,0309 & & 0,0000 & & 0,0008 & & 0,0300 & & 0,1419 & \\
\hline \multirow[t]{2}{*}{ inalen } & 0,2316 & $* *$ & 0,1229 & $* *$ & 0,0039 & & 0,0757 & $* *$ & 0,0129 & $*$ & 0,0110 & \\
\hline & 0,0261 & & 0,0160 & & 0,8237 & & 0,0150 & & 0,0515 & & 0,6105 & \\
\hline \multirow[t]{2}{*}{ inalol } & $-0,0661$ & $*$ & $-0,0471$ & $* *$ & $-0,0178$ & & $-0,0068$ & & 0,0470 & & 0,1043 & \\
\hline & 0,0542 & & 0,0364 & & 0,5932 & & 0,7477 & & 0,4777 & & 0,3661 & \\
\hline \multirow[t]{2}{*}{ cosden } & $-0,0056$ & $* * *$ & $-0,0037$ & $*$ & 0,0000 & & $-0,0055$ & $* *$ & $-0,0047$ & $* *$ & $-0,0054$ & $*$ \\
\hline & 0,0077 & & 0,0978 & & 0,9904 & & 0,0178 & & 0,0500 & & 0,0503 & \\
\hline \multirow[t]{2}{*}{ ros } & 0,0013 & & 0,0013 & & 0,0005 & & $-0,0003$ & & 0,0014 & & $-0,0024$ & \\
\hline & 0,3753 & & 0,3394 & & 0,6740 & & 0,8078 & & 0,2633 & & 0,1067 & \\
\hline \multirow[t]{2}{*}{ roe } & $-0,0001$ & & 0,0002 & & 0,0011 & $*$ & 0,0014 & $*$ & 0,0004 & & 0,0021 & $* * *$ \\
\hline & 0,8945 & & 0,6830 & & 0,0984 & & 0,0860 & & 0,6777 & & 0,0019 & \\
\hline \multirow[t]{2}{*}{ curr } & 0,0589 & $* * *$ & 0,0374 & $* * *$ & 0,0291 & $* * *$ & 0,0214 & $* * *$ & 0,0254 & $* * *$ & 0,0169 & $*$ \\
\hline & 0,0000 & & 0,0042 & & 0,0001 & & 0,0014 & & 0,0010 & & 0,0544 & \\
\hline \multirow[t]{2}{*}{ solven } & 0,0045 & & $-0,0297$ & & $-0,0272$ & & 0,0012 & & 0,0495 & & 0,0334 & \\
\hline & 0,9503 & & 0,6867 & & 0,5238 & & 0,9845 & & 0,4568 & & 0,5929 & \\
\hline \multirow[t]{2}{*}{ Idim } & 0,0060 & & 0,0167 & & 0,0003 & & $-0,0102$ & & $-0,0158$ & & $-0,0114$ & \\
\hline & 0,6278 & & 0,1660 & & 0,9775 & & 0,4222 & & 0,2044 & & 0,3721 & \\
\hline \multirow[t]{2}{*}{ logeta } & $-0,0177$ & & $-0,0304$ & $* *$ & $-0,0285$ & $* *$ & $-0,0329$ & $* *$ & $-0,0263$ & $*$ & $-0,0318$ & $* *$ \\
\hline & 0,1365 & & 0,0152 & & 0,0319 & & 0,0140 & & 0,0506 & & 0,0316 & \\
\hline darea & 0,0450 & & 0,0611 & $* *$ & 0,0443 & & 0,0508 & $*$ & 0,0619 & $*$ & 0,0553 & \\
\hline \multicolumn{13}{|l|}{ deforta } \\
\hline \multicolumn{13}{|l|}{ crclta } \\
\hline \multirow[t]{2}{*}{ Cons } & $-0,0072$ & & $-0,0292$ & & 0,1069 & & 0,2553 & $* *$ & 0,2225 & $*$ & 0,2408 & \\
\hline & 0,9551 & & 0,8119 & & 0,3834 & & 0,0439 & & 0,0927 & & 0,0903 & \\
\hline $\mathrm{N}$ & 455 & & 453 & & 452 & & 449 & & 455 & & 469 & \\
\hline $\mathrm{R}^{2}$ & 0,1195 & & 0,1027 & & 0,1287 & & 0,1915 & & 0,1375 & & 0,1382 & \\
\hline $\mathrm{F}$ & 4,7360 & & 3,6429 & & 4,8731 & & 7,5728 & & 7,7393 & & 5,9691 & \\
\hline
\end{tabular}

Level of significance: $\left({ }^{* * *}\right) 1 \% ;\left({ }^{* *}\right) 5 \% ;\left({ }^{*}\right) 10 \%$

Interdependence between management policies of trade receivables and payables is considered through the comparison between gross and net models, noting that the choices of recourse to trade receivables and payables are closely interrelated since the net models have constantly greater predictive power than gross models. 
Moving on to observe the results relate to the third research hypothesis, for the industrial sector (Tables no. 911) we find the absence of a constant relationship of dependency between the dependent variables and predictors of a financial nature in all years under observation. In particular, the incidence of trade payables (deforta) has statistically significant relationships with the incidence of short-term bank debt (inbanel) only in 2011 (Table no. 10). The incidence of trade receivables ( $c r c l t a)$ has statistically significant positive relationships with the incidence of short-term bank debt (inbanel) only since 2008 (Table no. 9): this suggests that transactional motivation in the use of trade credit are relevant to industrial firms especially in the years following the outbreak of the crisis.

Significant and negative relationships are observed between the dependent variables and the incidence of medium and long term bank debt (inbanol), except for the years 2006 and 2008 with respect to the variable deforta (Table no. 10) and 2011 for the variable crmerta (Table no. 11). These reports emphasize the importance of substitutability between trade credit and bank loans also for the industrial firms. Statistically significant relationships with other predictors of a financial nature do not show a similar constancy in the results, highlighting the importance of real type motivations for industrial sector. With respect to the impact of financial charges cosden, dependency relationships are present but not constant over the years. For the dependent variable deforta there is a positive and statistically significant relationship only in the first two years of observation (Table no. 10): the higher cost of money leads to an increased use of trade payables by firms operating in the industry and agriculture.

For variables crclta and cremerta (Tables no. 9 and 11) significance is observed especially in the last three years (2009-2011) and it is a negative relationship The higher cost of money in the years of crisis causes lower deferred payment and a minimization of the financial needs determined by the incidence of net mercantile credit.

Moving on to the analysis of the results attributable to companies that operate in the services sector (Tables no. 12 - 14), statistically, significant dependency relationships of the three dependent variables with the incidence of short-term bank debt (inbanen) are observed in all the six years. For crclta and crmerta the relationships are positive and reiterate the importance of motivation of transactional type in the use of trade credit (Tables no. 12 and 14); for deforta the relationships are negative manifesting conditions of substitutability that coexist with conditions of complementarity (Table no. 13). For the variables crclta and deforta there are also negative dependency relationships with the incidence of medium and long term bank debt (inbanol) and for the variable deforta there are negative dependency relationships with the incidence of debt to other financiers, indicating conditions of substitutability. 
Table 12: Regression Analysis 2006-2011 - Dependent Variable: crclta - Services

\begin{tabular}{|c|c|c|c|c|c|c|c|c|c|c|c|c|}
\hline & 2006 & & 2007 & & 2008 & & 2009 & & 2010 & & 2011 & \\
\hline \multirow[t]{2}{*}{ inbanen } & 0,0008 & $* * *$ & 0,0006 & $* * *$ & 0,0007 & $* * *$ & 0,0010 & $* * *$ & 0,0010 & $* * *$ & 0,0007 & $* * *$ \\
\hline & 0,0000 & & 0,0000 & & 0,0000 & & 0,0000 & & 0,0000 & & 0,0000 & \\
\hline \multirow[t]{2}{*}{ inbanol } & $-0,0217$ & $* * *$ & $-0,0126$ & & $-0,1037$ & $* * *$ & $-0,0587$ & $* * *$ & $-0,0318$ & & $-0,0401$ & $* *$ \\
\hline & 0,0019 & & 0,1146 & & 0,0000 & & 0,0055 & & 0,1153 & & 0,0406 & \\
\hline \multirow[t]{2}{*}{ inalen } & $-0,0224$ & & $-0,0999$ & $* * *$ & $-0,1223$ & $*$ & $-0,1959$ & & $-0,0604$ & & $-0,0072$ & \\
\hline & 0,1237 & & 0,0045 & & 0,0682 & & 0,2490 & & 0,7506 & & 0,9674 & \\
\hline \multirow[t]{2}{*}{ inalol } & $-0,0242$ & & 0,0104 & & 0,0544 & $* * *$ & 0,0946 & & $-0,0249$ & & $-0,0133$ & \\
\hline & 0,8168 & & 0,8517 & & 0,0028 & & 0,1520 & & 0,9054 & & 0,9525 & \\
\hline \multirow[t]{2}{*}{ cosden } & $-0,0030$ & & $-0,0062$ & $*$ & $-0,0005$ & & $-0,0040$ & & $-0,0080$ & $* *$ & $-0,0102$ & $* * *$ \\
\hline & 0,3768 & & 0,0570 & & 0,8984 & & 0,2866 & & 0,0440 & & 0,0021 & \\
\hline \multirow[t]{2}{*}{ ros } & $-0,0050$ & & $-0,0092$ & $* * *$ & $-0,0047$ & $*$ & $-0,0011$ & & $-0,0019$ & & $-0,0035$ & \\
\hline & 0,1839 & & 0,0007 & & 0,0759 & & 0,6509 & & 0,5527 & & 0,1165 & \\
\hline \multirow{2}{*}{ roe } & 0,0017 & $*$ & 0,0033 & $* * *$ & 0,0023 & $* * *$ & 0,0025 & $* * *$ & 0,0021 & $* *$ & 0,0022 & $* *$ \\
\hline & 0,0815 & & 0,0003 & & 0,0072 & & 0,0095 & & 0,0341 & & 0,0204 & \\
\hline \multirow[t]{2}{*}{ curr } & 0,0418 & & 0,1009 & $* * *$ & 0,1455 & $* * *$ & 0,1148 & $* * *$ & 0,1564 & $* * *$ & 0,1292 & $* * *$ \\
\hline & 0,1630 & & 0,0002 & & 0,0000 & & 0,0000 & & 0,0000 & & 0,0000 & \\
\hline \multirow[t]{2}{*}{ solven } & $-0,3260$ & $* * *$ & $-0,3982$ & $* * *$ & $-0,6930$ & $* * *$ & $-0,6317$ & $* * *$ & $-0,6788$ & $* * *$ & $-0,7527$ & $* * *$ \\
\hline & 0,0059 & & 0,0002 & & 0,0000 & & 0,0000 & & 0,0000 & & 0,0000 & \\
\hline \multirow[t]{2}{*}{ Idim } & $-0,0002$ & & 0,0081 & & $-0,0037$ & & $-0,0263$ & $*$ & $-0,0093$ & & $-0,0189$ & \\
\hline & 0,9879 & & 0,5727 & & 0,7937 & & 0,0827 & & 0,5452 & & 0,1759 & \\
\hline \multirow[t]{2}{*}{ logeta } & 0,0589 & $* *$ & 0,0676 & $* * *$ & 0,0431 & $* *$ & 0,0351 & & 0,0287 & & 0,0272 & \\
\hline & 0,0230 & & 0,0056 & & 0,0392 & & 0,1055 & & 0,2134 & & 0,2590 & \\
\hline \multirow[t]{2}{*}{ darea } & 0,1149 & $* * *$ & 0,1234 & $* * *$ & 0,1169 & $* * *$ & 0,0858 & $* *$ & 0,0592 & & 0,0520 & \\
\hline & 0,0058 & & 0,0009 & & 0,0033 & & 0,0272 & & 0,1633 & & 0,2081 & \\
\hline \multicolumn{13}{|l|}{ deforta } \\
\hline \multicolumn{13}{|l|}{ crclta } \\
\hline \multirow[t]{2}{*}{ Cons } & 0,1611 & & 0,0177 & & 0,2070 & & 0,4627 & $* * *$ & 0,3313 & $*$ & 0,5097 & $* * *$ \\
\hline & 0,3510 & & 0,9043 & & 0,1751 & & 0,0058 & & 0,0683 & & 0,0028 & \\
\hline $\mathrm{N}$ & 252 & & 258 & & 250 & & 258 & & 249 & & 251 & \\
\hline $\mathrm{R}^{2}$ & 0,1634 & & 0,2830 & & 0,3616 & & 0,3051 & & 0,3500 & & 0,3906 & \\
\hline $\mathrm{F}$ & 20,0939 & & 79,3962 & & 33,6142 & & 27,9318 & & 23,9239 & & 32,7222 & \\
\hline
\end{tabular}

Level of significance: $\left({ }^{* * *}\right) 1 \%$; $\left({ }^{* *}\right) 5 \%$; $\left({ }^{*}\right) 10 \%$ 
Table 13: Regression Analysis 2006-2011 - Dependent Variable: defientten-Senviimess

\begin{tabular}{|c|c|c|c|c|c|c|c|c|c|c|c|c|}
\hline & 2006 & & 2007 & & 2008 & & 2009 & & 2010 & & 2011 & \\
\hline \multirow[t]{2}{*}{ inbanen } & $-0,0002$ & $* * *$ & $-0,0003$ & $* * *$ & $-0,0004$ & $* * *$ & $-0,0004$ & $* * *$ & $-0,0003$ & $* * *$ & $-0,0005$ & $* * *$ \\
\hline & 0,0000 & & 0,0000 & & 0,0000 & & 0,0000 & & 0,0000 & & 0,0000 & \\
\hline \multirow[t]{2}{*}{ inbanol } & $-0,0142$ & $* * *$ & $-0,0074$ & $* *$ & $-0,0645$ & $* * *$ & $-0,0457$ & $* * *$ & $-0,0208$ & $*$ & $-0,0232$ & $* *$ \\
\hline & 0,0008 & & 0,0460 & & 0,0000 & & 0,0006 & & 0,0732 & & 0,0212 & \\
\hline \multirow[t]{2}{*}{ inalen } & 0,0222 & $* * *$ & 0,0312 & & $-0,1129$ & $* *$ & $-0,0647$ & & $-0,0065$ & & 0,0787 & \\
\hline & 0,0002 & & 0,6685 & & 0,0485 & & 0,2783 & & 0,9256 & & 0,4919 & \\
\hline \multirow[t]{2}{*}{ inalol } & $-0,0589$ & & $-0,1152$ & $* *$ & 0,0434 & $* * *$ & 0,0505 & $* *$ & $-0,1146$ & $*$ & $-0,2630$ & $* * *$ \\
\hline & 0,1999 & & 0,0428 & & 0,0010 & & 0,0414 & & 0,0839 & & 0,0062 & \\
\hline \multirow[t]{2}{*}{ cosden } & 0,0039 & $*$ & 0,0004 & & $-0,0025$ & & 0,0032 & & 0,0015 & & $-0,0010$ & \\
\hline & 0,0995 & & 0,8791 & & 0,3006 & & 0,1820 & & 0,5056 & & 0,6835 & \\
\hline \multirow[t]{2}{*}{ ros } & $-0,0081$ & $* * *$ & $-0,0102$ & $* * *$ & $-0,0070$ & $* * *$ & $-0,0024$ & & $-0,0012$ & & $-0,0061$ & $* * *$ \\
\hline & 0,0037 & & 0,0000 & & 0,0009 & & 0,2193 & & 0,5635 & & 0,0018 & \\
\hline \multirow[t]{2}{*}{ roe } & 0,0022 & $* * *$ & 0,0030 & $* * *$ & 0,0024 & $* * *$ & 0,0028 & $* * *$ & 0,0018 & $* *$ & 0,0023 & $* * *$ \\
\hline & 0,0075 & & 0,0000 & & 0,0006 & & 0,0001 & & 0,0120 & & 0,0012 & \\
\hline \multirow[t]{2}{*}{ curr } & $-0,0013$ & & 0,0204 & & 0,0401 & $* * *$ & 0,0321 & $* *$ & 0,0406 & $* * *$ & 0,0212 & \\
\hline & 0,9322 & & 0,1895 & & 0,0094 & & 0,0121 & & 0,0053 & & 0,1893 & \\
\hline \multirow[t]{2}{*}{ solven } & $-0,4506$ & $* * *$ & $-0,4857$ & $* * *$ & $-0,6494$ & $* * *$ & $-0,5848$ & $* * *$ & $-0,5871$ & $* * *$ & $-0,6061$ & $* * *$ \\
\hline & 0,0000 & & 0,0000 & & 0,0000 & & 0,0000 & & 0,0000 & & 0,0000 & \\
\hline \multirow[t]{2}{*}{ Idim } & $-0,0120$ & & $-0,0062$ & & $-0,0164$ & $*$ & $-0,0261$ & $* * *$ & $-0,0203$ & $* *$ & $-0,0174$ & $*$ \\
\hline & 0,2145 & & 0,5120 & & 0,0844 & & 0,0069 & & 0,0320 & & 0,0768 & \\
\hline \multirow[t]{2}{*}{ logeta } & 0,0199 & & 0,0171 & & 0,0062 & & 0,0162 & & 0,0095 & & 0,0115 & \\
\hline & 0,2500 & & 0,3299 & & 0,6973 & & 0,2829 & & 0,5441 & & 0,5142 & \\
\hline \multirow[t]{2}{*}{ darea } & $-0,0348$ & & $-0,0473$ & $*$ & $-0,0379$ & & $-0,0382$ & & $-0,0462$ & $*$ & $-0,0397$ & $*$ \\
\hline & 0,1743 & & 0,0912 & & 0,1182 & & 0,1109 & & 0,0514 & & 0,0832 & \\
\hline \multicolumn{13}{|l|}{ deforta } \\
\hline \multicolumn{13}{|l|}{ crclta } \\
\hline \multirow[t]{2}{*}{ Cons } & 0,5066 & $* * *$ & 0,4783 & $* * *$ & 0,6357 & $* * *$ & 0,6171 & $* * *$ & 0,5903 & $* * *$ & 0,6089 & $* * *$ \\
\hline & 0,0000 & & 0,0000 & & 0,0000 & & 0,0000 & & 0,0000 & & 0,0000 & \\
\hline $\mathrm{N}$ & 252 & & 258 & & 250 & & 258 & & 249 & & 251 & \\
\hline $\mathrm{R}^{2}$ & 0,3537 & & 0,3691 & & 0,4729 & & 0,4487 & & 0,4071 & & 0,4511 & \\
\hline $\mathrm{F}$ & 15,7842 & & 14,8011 & & 17,1104 & & 18,6308 & & 17,1678 & & 17,5950 & \\
\hline
\end{tabular}

Level of significance: $\left({ }^{* *}\right) 1 \% ;\left({ }^{* *}\right) 5 \% ;\left({ }^{*}\right) 10 \%$ 


\begin{tabular}{|c|c|c|c|c|c|c|c|c|c|c|c|c|}
\hline & 2006 & & 2007 & & 2008 & & 2009 & & 2010 & & 2011 & \\
\hline \multirow[t]{2}{*}{ inbanen } & 0,0010 & $* * *$ & 0,0010 & $* * *$ & 0,0011 & $* * *$ & 0,0014 & $* * *$ & 0,0013 & $* * *$ & 0,0012 & $* * *$ \\
\hline & 0,0000 & & 0,0000 & & 0,0000 & & 0,0000 & & 0,0000 & & 0,0000 & \\
\hline \multirow[t]{2}{*}{ inbanol } & $-0,0074$ & & $-0,0053$ & & $-0,0391$ & & $-0,0130$ & & $-0,0110$ & & $-0,0169$ & \\
\hline & 0,1782 & & 0,3329 & & 0,1281 & & 0,5706 & & 0,3653 & & 0,2255 & \\
\hline \multirow[t]{2}{*}{ inalen } & $-0,0447$ & $* * *$ & $-0,1312$ & $* *$ & $-0,0094$ & & $-0,1312$ & & $-0,0539$ & & $-0,0859$ & \\
\hline & 0,0002 & & 0,0163 & & 0,8727 & & 0,4430 & & 0,7578 & & 0,4899 & \\
\hline \multirow[t]{2}{*}{ inalol } & 0,0346 & & 0,1256 & & 0,0110 & & 0,0442 & & 0,0896 & & 0,2497 & \\
\hline & 0,7651 & & 0,1643 & & 0,5316 & & 0,4587 & & 0,6568 & & 0,2322 & \\
\hline \multirow[t]{2}{*}{ cosden } & $-0,0069$ & $*$ & $-0,0066$ & $*$ & 0,0020 & & $-0,0071$ & $*$ & $-0,0096$ & $* *$ & $-0,0091$ & $* * *$ \\
\hline & 0,0762 & & 0,0860 & & 0,6672 & & 0,0631 & & 0,0274 & & 0,0097 & \\
\hline \multirow[t]{2}{*}{ ros } & 0,0031 & & 0,0009 & & 0,0023 & & 0,0013 & & $-0,0008$ & & 0,0026 & \\
\hline & 0,2501 & & 0,6883 & & 0,4370 & & 0,3696 & & 0,6847 & & 0,2195 & \\
\hline \multirow[t]{2}{*}{ roe } & $-0,0005$ & & 0,0004 & & 0,0000 & & $-0,0002$ & & 0,0003 & & 0,0000 & \\
\hline & 0,6473 & & 0,7080 & & 0,9832 & & 0,8059 & & 0,7873 & & 0,9890 & \\
\hline \multirow[t]{2}{*}{ curr } & 0,0430 & $*$ & 0,0804 & $* * *$ & 0,1054 & $* * *$ & 0,0827 & $* * *$ & 0,1158 & $* * *$ & 0,1080 & $* * *$ \\
\hline & 0,0715 & & 0,0002 & & 0,0000 & & 0,0001 & & 0,0000 & & 0,0000 & \\
\hline \multirow[t]{2}{*}{ solven } & 0,1245 & & 0,0875 & & $-0,0436$ & & $-0,0470$ & & $-0,0917$ & & $-0,1466$ & $*$ \\
\hline & 0,2641 & & 0,3938 & & 0,6457 & & 0,5735 & & 0,2411 & & 0,0697 & \\
\hline \multirow[t]{2}{*}{ Idim } & 0,0118 & & 0,0142 & & 0,0127 & & $-0,0002$ & & 0,0110 & & $-0,0015$ & \\
\hline & 0,4241 & & 0,3257 & & 0,4183 & & 0,9906 & & 0,5060 & & 0,9162 & \\
\hline \multirow[t]{2}{*}{ logeta } & 0,0390 & & 0,0505 & $* *$ & 0,0369 & & 0,0189 & & 0,0192 & & 0,0157 & \\
\hline & 0,1502 & & 0,0412 & & 0,1238 & & 0,3887 & & 0,4140 & & 0,5144 & \\
\hline darea & 0,1497 & $* * *$ & 0,1706 & $* * *$ & 0,1548 & $* * *$ & 0,1241 & $* * *$ & 0,1054 & $* *$ & 0,0917 & $* *$ \\
\hline \multicolumn{13}{|l|}{ deforta } \\
\hline \multicolumn{13}{|l|}{ crclta } \\
\hline \multirow[t]{2}{*}{ Cons } & $-0,3455$ & $*$ & $-0,4606$ & $* * *$ & $-0,4288$ & $* *$ & $-0,1543$ & & $-0,2590$ & & $-0,0993$ & \\
\hline & 0,0567 & & 0,0066 & & 0,0107 & & 0,3885 & & 0,1832 & & 0,5614 & \\
\hline $\mathrm{N}$ & 252 & & 258 & & 250 & & 258 & & 249 & & 251 & \\
\hline $\mathrm{R}^{2}$ & 0,1791 & & 0,2303 & & 0,1975 & & 0,1655 & & 0,2034 & & 0,2130 & \\
\hline $\mathrm{F}$ & 25,2224 & & 22,3329 & & 19,2845 & & 14,4897 & & 12,0782 & & 24,6782 & \\
\hline
\end{tabular}

Level of significance: $\left({ }^{* * *}\right) 1 \% ;\left({ }^{* *}\right) 5 \% ;\left({ }^{*}\right) 10 \%$

Finally, it is interesting to note that for the variable deforta is absent a statistically significant relationship with the impact of financial charges (cosden) in most years under analysis. The cost of money does not affect the recourse to trade payables, even in this case also underlying the importance of motivations of real type in the use of trade payables for service firms.

\section{CONCLUSION}

For many Italian firms during the crisis, financial needs increased because of the growth of the working capital. Payment deadlines were extended for commercial transactions and this generated greater difficulty in maintaining a financial equilibrium, especially in SMEs with less bargaining power (Banca d'Italia 2010, 2011, 2012, 2013), confirming the importance of trade credits with real motivations, but also transactional reasons and financial reasons. Firms that have reduced access to bank debt may be forced to use trade credit more frequently than firms which are not subject to credit rationing. On the other hand, trade credit improves the visibility and reputation of the borrowing firms, making the banks more open to granting finance to healthy 
projects that they would have otherwise refused. A dynamic and complementary relationship exists between trade credit and bank financing, which results in partial substitutability. Enterprises use trade credit because they are exposed to credit rationing - in this a substitution effect is formed -and at the same time trade credit signalises and reveals information to the banks about the reliability of the firms receiving payment deferrals, facilitating access to bank debt.

The results of the paper highlight the existence of an interdependent relationship between trade credit policy and trade debt policy in SMEs (first hypothesis), and the coexistence of conditions of complementarity and substitutability between trade credit and other financing sources (second and third hypothesis). The results of the analysis referring to the incidence of trade payables signal a substitutability relationship between intercompany debt and financing from banks or third parties. The hypotheses of the coexistence of conditions of complementarity and substitutability is verified by observing the results referring to the incidence of trade credit. We can see a direct functional relationship between the incidence of trade credit and the incidence of short-term bank financing, testifying double intermediary conditions and a complementarity between the two variables. The importance of transactional motivations has been highlighted.

Regarding the variable which represents the incidence of net mercantile credit, we can also confirm the coexistence of complementarity and substitutability conditions. The empirical analysis shows a significant direct relationship between the dependent variable and short-term bank financing and an inverse relationship with the incidence of medium and long-term bank financing and short-term financing from third parties.

The direct relationship between the incidence of trade payables and the incidence of financial charges reiterates the importance of substitutability in the years preceding the crisis. While during the crisis (20082011) the inverse relationship between the incidence of trade receivables and the incidence of financial charges reduces the convenience of double intermediation. Conditions of substitutability and complementarity can also be observed by considering separately the sample firms involved in agricultural or industrial activities and the sample firms that perform services. For both categories, we observe the coexistence of complementarity and substitutability, but in the case of industrial firm complementarity conditions and the relief of the transactional reasons are observed over the crisis years, from 2008 onwards, and not in the early years of the analysis. Significant relationships with other predictors of a financial nature do not show a similar constancy in the results, highlighting the importance of real type motivations for the industrial sector.

In the case of service companies, however, relations with the predictors of financial nature are weakened during the crisis years, reaffirming the importance of the motivations of real type in the use of trade credit. In keeping with reference literature (inter alia: Alphonse Ducret and Severin 2004), the results of the study allow us to state that substitutability and complementarity between trade credit, bank financing and financing from third parties can be verified in the same financial-economic system, without having to exclude each other. The results of the study also allow to state that the substitutability and/or complementarity conditions can operate together with the prevailing of real motivations of recourse in trade credit. In financial systems characterized by the presence of SMEs and in situations of economic and financial crisis the close interconnection between real and financial motivations for recourse in intercompany credit is therefore reiterated. 


\section{REFERENCES}

Alphonse, P. Ducret, J. Severin, E. 2004, "When trade credit facilitates access to bank finance: Evidence from US small business data", EFMA Base Meetings Paper.

Banca d'Italia Economic Bulletin, various years, Roma.

Banca d'Italia Regional economies, various years, Roma.

Banca d'Italia, Annual Report, various years, Roma.

Biais, B. Gollier, C. 1997, "Trade Credit and Credit Rationing", Review of Financial Studies, vol. 10, No. 4, pp. $903-937$.

Cannari, L. Chiri, S. Omiccioli, M. 2004, "Condizioni del credito commerciale e differenziazione della clientela", Banca d'Italia, Temi di discussione, No. 495.

Cannari, L. Chiri, S. Omiccioli, M. 2005, Imprese o intermediari? Aspetti finanziari e commerciali del credito tra imprese in Italia, II Mulino.

Carmignani, A. 2004, Funzionamento della giustizia civile e struttura finanziaria delle imprese: il ruolo del credito commerciale, Banca d'Italia, Temi di discussione, No. 497.

Demirguc-Kunt, A. Maksimovic, V. 2001, Firms as Financial Intermediaries: evidence from trade credit data, World Bank Policy Research Working Paper No. 2696, World Bank Development Research Group (DECRG) and University of Maryland.

Duca, J. 1986, "Trade credit and credit rationing: a theoretical model", Board of Governors of the Federal Reserve System, Research Papers in Banking and Financial Economics, No. 94.

Emery, G.W. 1987, “An optimal financial response to variable Demand”, Journal of Financial and Quantitative Analysis, Vol. 22, pp. 209225.

Emery, G.W. 1984, “A pure financial explanation for trade credit”, Journal of Financial and Quantitative analysis, Vol. 19, pp. 271-285.

Fabbri, D. Klapper, L. 2008, "Market power and the matching of trade credit terms”, World Bank Policy research working paper, no. 4754.

Ferris, J.S. 1981, “A transactions theory of trade credit use”, Quarterly journal of Economics, vol. 96, no. 2 pp. $243-270$.

Finaldi Russo, P. Leva, L. 2004, “Il debito commerciale in Italia: quanto contano le motivazioni finanziarie?” Temi di discussione, No. 497, Banca d'Italia, Roma.

Fisman, R. Love, I. 2002, "Trade credit, financial intermediary development and industry growth", NBER working paper, No 8960.

Forestieri G. 1975, Il credito mercantile, Giuffrè.

Fox, J. 1997, Applied regressions analysis, linear models, and related methods, Sage Publications, USA.

Gibilaro, L. Mattarocci, G. 2010, "Le variabili predittive della posizione netta in credito commerciale: evidenze empiriche per le imprese italiane", Credifact, Discussion Paper Series, No. 1.

Huyghebaert, N. 2006, "On the Determinants and Dynamics of Trade Credit: Empirical Evidence from Business Start-ups", Journal of Business Finance \& Accounting, Vol. 33, pp. 305-328.

Jaffe, D.M. Stiglitz, J.E. 1990, Credit rationing, in Friedman B.M. e Hahn F.H., (Ed.), Handbook of Monetary Economics, Amsterdam, North Holland.

Lee, Y.W. Stowe, J.D. 1993, "Product risk, asymmetric information, and trade credit", Journal of Financial and Quantitative Analysis, Vol. 28, No. 2, pp.88-96.

Long, M.S. Malitz, I.B. Ravid, S.A. 1993, "Trade Credit, Quality guarantees, and Product Marketability", Financial Management, Vol. 22, No. 4, pp. 117-127.

Mian, S.L. e Smith, C.W. 1992, “Accounts receivable policy management: theory and evidence”, Journal of Finance, vol. 47, pp. 169-200.

Panetta 2003, "Evoluzione del sistema bancario e finanziamento dell'economia nel Mezzogiorno", Banca d'Italia, Temi di Discussione, No. 467.

Petersen, M.A. Rajan, R.G. 1997, "Trade credit: theories and evidence”, The Review of Financial Studies, Vol. 10, pp. 661-691.

Pike R., Nam Sang Cheng, Cravens K. 2005, "Trade Credit Terms: Asymmetric Information and Price Discrimination Evidence From Three Continents", Journal of Business Finance \& Accounting, Vol. 32, pp. 1197-1236.

Schwartz, R.A. 1974, "An economic model of trade credit", Journal of Financial and Quantitative Analysis, Vol. 9, pp. 643-657.

Schwartz, R.A. Whitcomb, D.K. 1978, "Implicit transfers in the extension of trade credit", in K.E. Boulding e T.F. Wilson (Ed.), Redistribution through the Financial System, New York.

Schwartz, R.A. Whitcomb, D.K. 1979, "The trade credit decision”, in Bicksler J. (Ed.), Handbook of financial economics, Amsterdam.

Stiglitz, J.E. Weiss, A. 1981, “Credit Rationing in Markets with Imperfect Information”, American Economic Review, Vol. 71, pp. $393-410$. 